\title{
Population-based study on axial ocular dimensions and corneal astigmatism
}

Khairidzan Mohd Kamal

International Islamic University Malaysia, Kuantan, Malaysia

As cataract surgery has evolved over 50 years, there have been changes in the current clinical ophthalmic practice to improve safety and efficacy in order to achieve perfect postoperative outcomes. Since the 1990s, technological advances such as ocular biometry, phacoemulsification, and intraocular lens (IOL) formulas, as well as many others, have undergone numerous incremental upgrades aiming to produce predictable and reproducible quality results. However, the financial burden of adopting all these proven yet costly technologies in ophthalmological practice, especially in rural areas, has to be justified. In this issue, an epidemiological study on ocular biometrics conducted by the Ministry of Health at the Kuala Pilah Cluster Program has shed some light on a possible strategy to incorporate advanced tools and technology into our public service.

As suggested by the findings from the National Eye Survey II study, untreated cataract accounts for more than half the cases of blindness in our country. ${ }^{1}$ The prevalence of cataract among the population 60 years of age and over verifies that the screening program should be targeted to this age group. Although this is not a novel finding, this hospital-based, cross-sectional study could well be the evidence that the current screening program has achieved significant mileage in addressing the issue. Loss of opportunity in detecting cataract can be reduced further if the screening program is carried out at every possible occasion. Improvements to the existing screening program should then be implemented internally to other departments in the public hospitals targeting the same age group.

The rapid progress in the advancement of IOL formulas and calculations can add to the complexity and ambiguity among surgeons in choosing their IOL. It has been proven that most of the available formulas provide adequate and predictable results to average axial length between 22 and $24 \mathrm{~mm} .{ }^{2}$ The relevancy of the SRKT formula usage in the majority of public institutions in Malaysia has now been substantiated by this study. The need for optical biometer measurements to incorporate the latest IOL formulas into the public practice may not be the highest priority at the moment. Utilization of the appropriate nomogram-based formula to deal with shorter or 
longer eyes in public practice is more practical and cost effective.

The incidence of corneal astigmatism among cataract patients has been well documented. ${ }^{3}$ More than a third of patients from Kuala Pilah cluster study found to have corneal astigmatism of more than 1D based only on anterior surface curvature measurement. If these data were subjected to the latest IOL formula calculations, nomograms or measurements that incorporate the effect of posterior corneal curvature, it is expected that more than half of this population would require astigmatic correction during cataract surgery4. The paradigm shift of targeting postoperative visual outcome from clarity of vision to spectacle independence and better quality vision has slowly infused in the public health system. As we are aiming to achieve postoperative spectacle freedom for our patients, this data highlighted that the need to invest on the training and proper equipment to address the corneal astigmatism management issue among cataract population.

The Kuala Pilah Cluster Cataract study was designed to look at ocular biometry epidemiological data. The results in this study might provide normative data for cataract patients and a useful reference for multiple purposes. It does suffer from few limitations but the value of its findings certainly go beyond establishing the normative data for clinical usage. It will be used to guide and help the Ministry of Health and other relevant authorities to direct our screening, training as well as cataract facility developmental program accordingly.

\section{References}

1. Chew FLM, Salowi MA, Mustari Z, et al. Estimates of visual impairment and its causes from the National Eye Survey in Malaysia (NESII). PloS One, 2018;13(6):e0198799.

2. Plat J, Hoa D, Mura F, et al., Clinical and biometric determinants of actual lens position after cataract surgery. J Cataract Refract Surg. 2017;43(2):195-199.

3. Cui, Y., et al., Biometry and corneal astigmatism in cataract surgery candidates from Southern China. J Cataract Refract Surg. 2014;40:1661-1669.

4. Reitblat O, Levy A, Kleinmann G, Abulafia A, Assia El. Effect of posterior corneal astigmatism on power calculation and alignment of toric intraocular lenses: comparison of methodologies. J Cataract Refract Surg. 2016;42:217-225. 